\title{
HBx and SP1 upregulate DKK1 expression
}

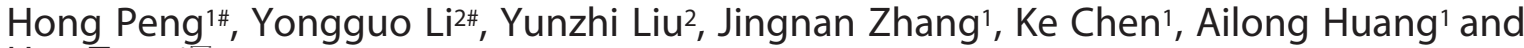 \\ Hua Tang 1
}

'Key Laboratory of Molecular Biology for Infectious Diseases (Ministry of Education), Institute for Viral Hepatitis, Department of Infectious Diseases, The Second Affiliated Hospital, Chongqing Medical University, Chongqing, PR China; '2Department of forensic medicine, Chongqing Medical University, Chongqing, 400016, China

\begin{abstract}
Numerous evidences suggested that the hepatitis B virus (HBV) was recognized as an important factor in the development of hepatocellular carcinoma (HCC). Dickkopf-1 (DKK1) recently was reported to be involved in the progress of HCC. HBV may regulate DKK1 expression in hematoma carcinogenesis. Here, we demonstrated that HBV could regulate DKK1 promoter activity which resulted in upregulation of its MRNA and protein expression in several HBV existing cell lines, and HBx played a prominent role in this process. Transcription factor binding site search result showed that there is a SP1 site in DKK1 promoter region. Luciferase assay showed that overexpression of SP1 could increase DKK1 promoter activity in a dose dependent manner. Accordingly, siRNA inhibition of SP1 expression reduced DKK1 promoter activity and decreased the expression of DKK1 protein.
\end{abstract}

Key words: HCC, HBV, DKK1, SP1

Received: 14 January, 2016; revised: 20 May, 2016; accepted: 13 July, 2016; available on-line: 10 October, 2016

e-mail: tanghua86162003@cqmu.edu.cn

\#Hong Peng and Yongguo Li contributed equally to this work.

Abbreviations: DKK1, Dickkopf-1; HBV, hepatitis B virus; HCC, hepatocellular carcinoma

\section{INTRODUCTION}

The mortality rate of HCC is the fourth highest worldwide (Schutte et al., 2009; El-Serag \& Rudolph, 2007). It has been reported that HBV played an important role in occurrence and development of HCC (Cougot et al., 2005). Previous reports showed that HBV could affect the progression of HCC by regulating the activity of gene promoters (Ling et al., 2013; Xu et al., 2014). The HBV genome consists of four overlapping open reading frames: $\mathrm{HBp}, \mathrm{HBs}, \mathrm{HBc}$ and $\mathrm{HBx}$ (Zhou et al., 2010). Now, more and more evidences available support the assumption that $\mathrm{HBx}$ and $\mathrm{HBs}$ protein have pathogenetic role in HCC. Especially, HBx was regarded as a promiscuous trans-activator of viral and cellular promoters and enhancers ( $\mathrm{Ng}$ \& Lee, 2011). HBx protein is a multifunctional regulator that modulates cellular signal transduction pathways, transcriptional regulations, cell cycle progression and DNA repair and apoptosis, which suggests that $\mathrm{HBx}$ plays a significant role in $\mathrm{HCC}(\mathrm{Ng} \&$ Lee, 2011; Keng et al., 2011).

Dickkopf-1, the prototype of a family of secreted proteins, plays a crucial role in head formation in vertebrate development and suppresses the progression of Wnt signaling (Glinka et al., 1998; Krupnik et al., 1999; Fedi et al., 1999; MacDonald et al., 2009). It acts as a tumor suppressor in colon tumors. However, overexpression of DKK1 was found in HCC and Wilms' tumor (GonzálezSancho et al., 2005; Wirths et al., 2003; Forget et al., 2007). In addition, it was reported that DKK1 could promote invasion and metastasis of hepatocelluar carcinoma (Tao et al., 2013). Whether HBV induced HCC through DKK1 pathway remains unknown, though. Here we reported that HBV elevated DKK1 expression in hepatoma carcinoma cells. Moreover, transcription factor SP1 intensified DKK1 expression upregulation by $\mathrm{HBV}$ by enhancing its promoter activity.

\section{MATERIALS AND METHODS}

Cell lines. Human hepatocarcinoma cell lines HepG2 and SMMC7721 were purchased from American Type Culture Collection, HepG2.2.15 (HBV expressing cells) AD38 cell line was kept by our laboratory. All HCC cell lines were cultured in DMEM (Hyclone, China) with 10\% FBS (Gibco, USA), 100U/ml penicillin and streptomycin, $5 \mathrm{mmol} / \mathrm{L}$ glutamine. Cells were grown in a $37^{\circ} \mathrm{C}, 5 \% \mathrm{CO}_{2}$ incubator. Transfections were performed with Lipofectamine 2000 (Invitrogen, USA) according to the manufacturer's instructions.

Total RNA isolation and quantitative real-time PCR. 48 hours after transfection, total RNA was extracted using TRIzol Reagent (Invitrogen, USA), and 2 $\mu \mathrm{g}$ of total RNA was used to synthesize cDNA with a PrimeScript RT Reagent Kit (Takara, Japan) according to the manufacturer's protocol. UltraSYBR mixture (Cwbio, China) was used in QRT-PCR to detect the expressions of gene on mRNA levels. The gene expression changes were calculated with the $2-\Delta \Delta \mathrm{CT}$ method (Livak et al., 2001). The primers used were as follows, $\beta$-actin-F: 5'-CСTTCTACAATGAGCTGCGT-3'; $\quad \beta$-actin-R: 5'-CCTGGATAGCAACGTACATG -3', DKK1-F: 5'-ACAGAGGCAGGGAAATGATAC-3'; DKK1-R: 5'-CAGCT'T'TCTCCTCCTCACATTC-3'; SP1-F: 5'-CTGAAGCTGGGTAGCCTATTG-3'; SP1-R: 5'-CTACTGCTGCGACCT'T'TCT'T-3'.

Construction of vectors. The pCMV-Sport6-HBx, pCMV-Sport6-HBs, pCMV-Sport6-HBc and pCMVSport6-HBp plasmids were constructed and their expression was confirmed as reported previously (Tian et al., 2011). During generation of the DKK1 promoter reporter plasmid pGL3-Basic-DKK1-P-wt, the HepG2 cell genomic DNA was used as template to amplify in PCR reaction, the sequence of DKK1 promoter was obtained from the Eukaryotic Promoter Database (http://epd. vital-it.ch/) and Regulatory Element Database (http:// rulai.cshl.edu/cgi-bin/TRED/tred.coiprocess=home). The primers were used as follows, pGL3-Basic-DKK1- 
P-F: 5'-ACGCGTCTGCCTAATCAAGT-3'; pGL3-Basic-DKK1-P-R: 5'-AATGACCGTCACT'TTGCAAG-3'. The mutant DKK1 promoter report plasmid, pGL3Basic-DKK1-P-mut-F, with removed the sequence containing SP1 binding sites, was generated based on pGL3-Basic-DKK1-P-wt plasmid by mutagenesis. The following primers were used, pGL3-Basic-DKK1-Pmut-F: 5'-CGAGCGACTAAGCAAGGGAGCCCATCCCGGCT'TTGTTGTC-3', pGL3-Basic-DKK1-P-mut-F: 5'-GACAACAAAGCCGGGATGGGCTCCCT'TGCTTAGTCGCTCG-3'.

Analysis of RNA interference (RNAi). The target sequence against mRNA of SP1 was SP1-siRNA: AGCGCTTCATGAGGAGTGA (Liu et al., 2006), the scrambled sequence GCT'TCATAAGGCGCATAGC was used as the control. These sequences were synthesized by Invitrogen.

Western blot analysis. Proteins were collected with RIPA buffer supplemented with $1 \mathrm{mmol} / \mathrm{L}$ PMSF. Protein concentration was determined with the BCA Assay Kit (Beyotime, China). Equal amounts of total protein was separated by $12 \%$ SDS-PAGE and then transferred to PVDF membranes. Those membranes were blocked in 5\% TBST with $0.5 \%$ Tween 20 for 2 hours at room temperature and incubated in primary antibody overnight at $4^{\circ} \mathrm{C}$. Membranes were then incubated with secondary antibody conjugated with HRP (diluted 1:4000) for 2 hours and washed in TBST three times. Then the signal was detected with a chemiluminescence ECL ${ }^{\mathrm{TM}}$ detection system (Pierce, USA).

Luciferase reporter assay. 48 hours after transfection, cells were collected, treated with $1 \times$ passive lysis buffer (PLB), and then gently shaken for $15 \mathrm{~min}$ at room temperature. Luciferase activity analysis was performed and normalized to the renilla luciferase using the luciferase assay kit (Dual-Luciferase Reporter Assay System, Promega, USA). All experiments were repeated at least 3 times.

Statistical analysis. Data were presented as mean value with standard deviation (S.D.). The independent $t$ test was used in statistical analysis. $P$ value less than 0.05 was considered statistically significant.

\section{RESULTS}

\section{HBV upregulated DKK1 expression}

To ascertain whether HBV could influence expression of DKK1, DKK1 expressions in HepG2 and HepG2.2.15 cells (expressing HBV) were measured with QRT-PCR and Western blot analysis. Our data showed that DKK1 had a higher expression in HepG2.2.15 cells than that in HepG2 cells (Fig. 1A, B). To further confirm the effect of HBV on DKK1 expression, we detected DKK1 expression in another HBV expressing AD38 cells with QRT-PCR and Western blot, AD38 cells with tetracycline used as a control (no HBV expression ), and as expected, DKK1 expression was upregulated by HBV in AD38 cells in comparison to that in AD38 cells induced by tetracycline (Fig. 1C, D). In addition, HepG2 cells infected with recombinant adenovirus expressing $\mathrm{HBV}$ (Ad-HBV) or its control GFP (Ad-GFP) were analyzed; results showed that the expression of DKK1 were obviously higher in Ad-HBV-HepG2 cells compared to the control Ad-GFP-HepG2 cells (Fig. 1E, $\mathrm{F})$. All these results suggested that HBV could upregulate the expression of DKK1.
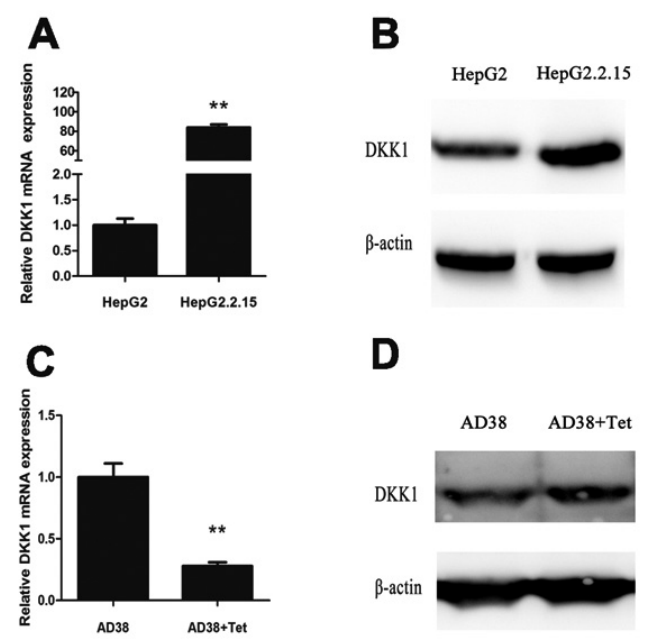

D

E
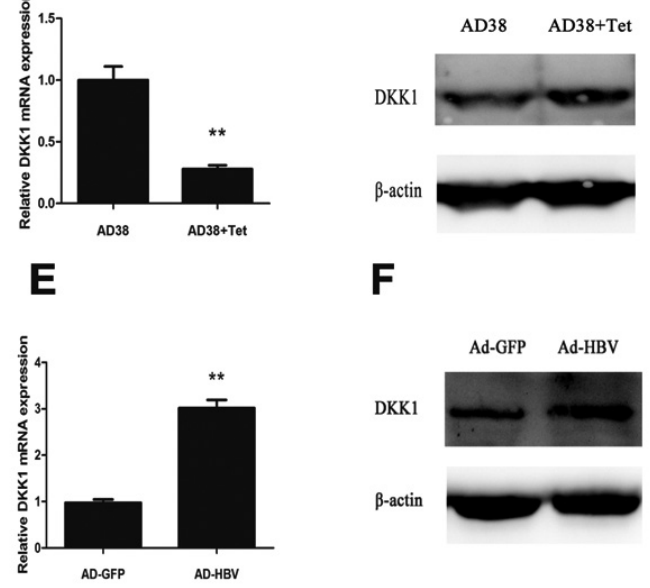

Figure. 1 Effects of HBV on the expression of DKK1.

(A) DKK1 mRNA expression in HepG2 and HepG2.2.15 cells was assessed with qRT-PCR, $\beta$-actin was used as control. (B) DKK1 protein expression in HepG2 and HepG2.2.15 cells was assessed with Western blot analysis. (C, D) DKK1 expression was determined in AD38 cells with Tet or AD38 cells. (E, F) DKK1 expression was analyzed in HepG2 cells which were transfected with Ad-GFP-HBV or Ad-GFP. ${ }^{*} P<0.01$

\section{HBx up-regulated DKK1 expression by improving its promoter activity}

To reveal how HBV modulates DKK1 expression, DKK1 promoter activity was measured with dual-luciferase reporter assays. First, we constructed pGL3Basic-DKK1-P-wt plasmid, which contained a luciferase gene under the control of a 899 bp DNA fragment in the proximal promoter region of DKK1. Next, pGL3Basic-DKK1-P-wt was transfected into HepG2 cells and HepG2.2.15 cells. Luciferase activity detemination showed that DKK1 promoter activity was significantly higher in HepG2.2.15 cells than that in HepG2 cells (Fig. 2A). The DKK1 promoter activity was enhanced in AD38 cells compared to that in AD38 cells with tetracycline when pGL3-Basic-DKK1-P-wt was used for transfection (Fig. 2B). Similarly, we found that DKK1 promoter had higher activity in Ad-HBV-HepG2 cells infected with Ad-HBV than in Ad-GFP-HepG2 cells transduced with Ad-GFP (Fig. 2C). Taken together, these results showed that HBV could upregulate DKK1 expression by increasing its promoter activity.

$\mathrm{HBV}$ encode four major proteins, $\mathrm{HBx}, \mathrm{HBc}, \mathrm{HBp}$ and HBs. To discover which one played a major role, the expression plasmids of these four major HBV proteins (pCMV-Sport6-HBx, pCMV-Sport6-HBc, pCMV-Sport6-HBp and pCMV -Sport6-HBs) were used for co-transfection with pGL3-Basic-DKK1-P-wt of SMMC7721 cells. We found that $\mathrm{HBx}$ could upregulate DKK1 promoter activity, while $\mathrm{HBc}, \mathrm{HBp}$ and $\mathrm{HBs}$ had no obvious influence on it (Fig. 2D). Furthermore, pCMV-Sport6-HBx, pCMV-Sport6-HBc, pCMV-Sport6$\mathrm{HBp}$ and pCMV-Sport6- HBs were respectively used for 
A

B

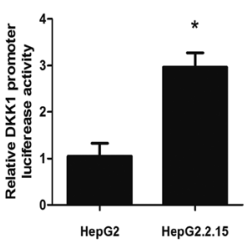

.

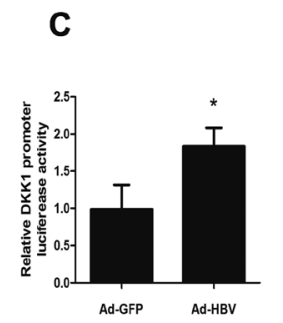

E

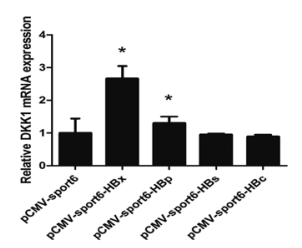

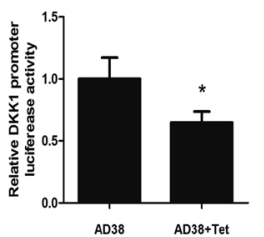

D

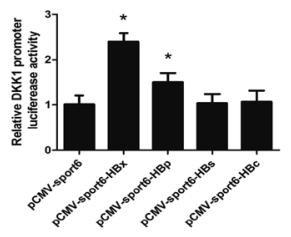

$\mathbf{F}$

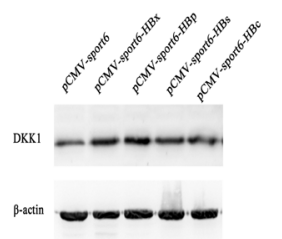

Figure. 2 The activity of DKK1 promoter was elevated by HBx. (A) pGL3-Basic-DKK1-P was used to transfect HepG2 cells and HepG2.2.15 cells. Then the activity of DKK1 promoter was measured with dual luciferase reporter assay. (B) The activity of DKK1 promoter was elevated in AD38 cells compared to AD38 cells with Tet. (C) The activity of DKK1 promoter was higher in HepG2 cells with Ad-HBV infection compared to Ad-GFP infection. (D) $0.3 \mu \mathrm{g}$ pGL3-Basic-DKK1-P, $0.1 \mu \mathrm{g}$ pRL-TK and pCMV-Sport6-HBx, pCMVSport6-HBC, pCMV-Sport6-HBp and pCMV-Sport6-HBs were used to transfect SMMC7721 cells. Dual-luciferase reporter assays were performed to detect DKK1 promoter activity. (E) SMMC7721 cells were co-transfected with $0.8 \mu \mathrm{g}$ pCMV-Sport6-HBx, pCMV-Sport6$\mathrm{HBC}, \mathrm{pCMV}$-Sport6-HBp and pCMV-Sport6-HBs, qRT-PCR was performed to detect DKK1 mRNA expressions. (F) After the same operation as in E, DKK1 protein expression was determined analyzed with Western blot. ${ }^{*} P<0.05$.

transfection of SMMC7721 cells, and the DKK1 expression was determined with qRT-PCR and Western blot analysis; we found that HBx played a significant role in upregulation of DKK1 expression by HBV (Fig. 2E, F).

\section{The transcription factor SP1 upregulated the expression of DKK1}

In order to search transcription factor binding sites in DKK1 promoter sequences, the following software was used, TFSEARCH (http://www.cbrc.jp/research/db/ TFSEARCH.html), Gene-Regulation (http://www. gene-regulation.com/pub/programs.html\#alibaba2). As a result, putative binding sites for transcription factors were found. To find out the decisive transcription factors which could regulate DKK1 promoter, pGL3-BasicDKK1-P-wt was used for co-transfection with different kinds of plasmids encoding the transcription factors, including pCMV-Sport6-NF- $x$ A, pCMV-Sport6-NF$x \mathrm{~B}, \mathrm{pCMV}-$ Sport6-NF- $x \mathrm{C}, \mathrm{pCMV}-$ Sport6-YY1, pCMVSport6-AP2 $\alpha$, and pCMV-Sport6-SP1, of the SMMC7721 cells. The results showed that only transcription factor SP1 could upregulate DKK1 promoter activity, while the rest of the transcription factors had no effects on it (Fig. 3A, and not shown), therefore we focused on SP1. We detected DKK1 promoter activity in SMMC7721

A

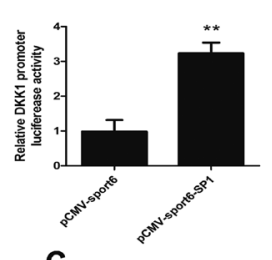

C
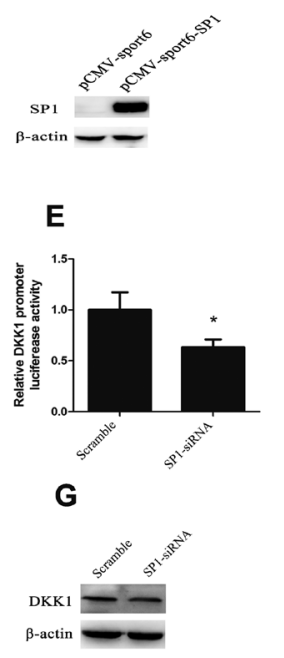

B

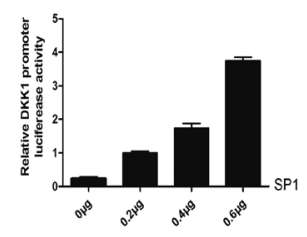

D

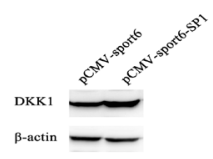

$\mathbf{F}$

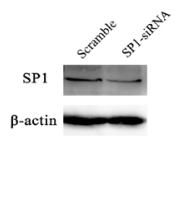

Figure. 3 Tanscription factor SP1 increased DKK1 expression.

(A) DKK1 promoter activity was determined after pGL3-Basic-DKK1-P, pRL-TK and PCMV-Sport6-SP1 co-transfection of SMMC7721 cells. pCMV-Sport6 was used as a control. (B) DKK1 promoter activity was analyzed after SMMC7721 cells were transiently transfected with different amounts of pCMV-Sport6-SP1 $(0 \mu \mathrm{g}, 0.2 \mu \mathrm{g}, 0.4 \mu \mathrm{g}, 0.6 \mu \mathrm{g})$. (C) SP1 expression in SMMC7721 cells after transfection with pCMV-Sport6-SP1. (D) DKK1 expression in SMMC7721 cells was checked after transfection with pCMVSport6 or pCMV-Sport6-SP1. (E) DKK1 promoter activities were analyzed after SMMC7721 cells were transfected with SP1-siRNA, scramble RNA were used as negative control. (F) The interference efficiency of SP1 was measured by Western blot. (G) DKK1 expression in SMMC7721 cells was checked after transfection with SP1SiRNA.

cells which were co-transfected with pGL3-Basic-DKK1P-wt and different amounts of SP1 $(0 \mu \mathrm{g}, 0.2 \mu \mathrm{g}, 0.4 \mu \mathrm{g}$, $0.6 \mu \mathrm{g}$, respectively) and measured it with dual-luciferase reporter assay (Fig. 3B). SP1 increased DKK1 promoter activity in a dose dependent manner. Then, the expression of DKK1 at protein level in SP1-overexpressing SMMC7721 cells was measured. In comparison to the control, overexpressed SP1 elevated DKK1 expression (Fig. 3D). To further confirm this result, PGL3Basic-DKK1-P-wt was used for co-tranfection of the SMMC7721 cells together with SP1-siRNA, which disturbed SP1 expression, and with scramble. As a result, DDK1 expression decreased both in DKK1 promoter activity and on protein level (Fig. 3E, 3F, 3G). Taken together, these results suggested that transcription factor SP1 could raise DKK1 expression by enhancing its promoter activity.

\section{Transcription factor SP1 and HBV both regulate the expression of DKK1}

The results above showed that HBV and SP1 could both upregulate DKK1 expressions by enhancing its promoter activity. We also explored the correlation between HBV and SP1 in regulating DKK1 expression. First, DKK1 promoter activities were checked in HepG2 
A

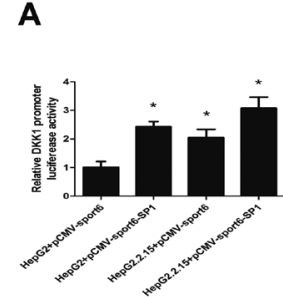

C

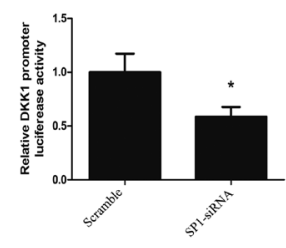

E

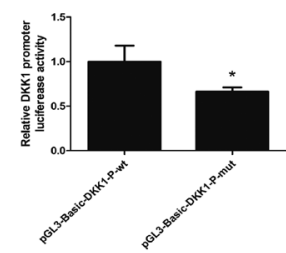

B

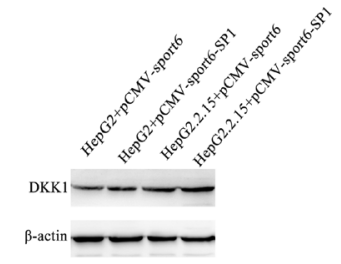

D

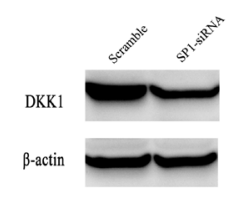

Figure. 4 SP1 assisted HBV regulating DKK1 expression.

(A) Relative DKK1 promoter activity in HepG2 and HepG2.2.15 cells which were transfected with pCMV-Sport6 or pCMV-Sport6-SP1, respectively. (B) Relative DKK1 protein expression was detected in cells as in A. (C) Relative DKK1 promoter activity in HepG2.2.15 cells transfected with scramble or 0 SP1-siRNA. (D) Relative DKK1 protein expression detected in HepG2.2.15 cells treated as in C. (E) Relative DKK1 promoter activity in HepG2.2.15 cells transfected with pGL3-Basic-DKK1-P-WT or pGL3-Basic-DKK1-P-Mut.

cells and HepG2.2.15 cells (HBV expressing cells) which were cotransfected with PGL3-Basic-DKK1-P-wt and pCMV-Sport6-SP1, respectively. It was found that SP1 could strikingly increase DKK1 promoter activity (Fig. 4A) and DKK1 protein expression (Fig. 4B) in presence of $\mathrm{HBV}$, whereas, with SP1 expression inhibited in HepG2.2.15 cells promoter activity of DKK1 was reduced (Fig. 4C), and protein expression of DKK1 also was downregulated (Fig. 4D). Secondly, for analysis of the SP1 function, pGL3-Basic-DKK1-P-mut was generated, in which SP1 binding sites was mutated, and then the plasmid was used for transfection of HepG2.2.15 cells, and pGL3-Basic-DKK1-P-wt was used as a control. DKK1 promoter activity was restrained as a result of loss of SP1 binding sites (Fig. 4E). This results revealed that SP1 cooperated with HBV to regulate DKK1 expression.

\section{DISCUSSION}

It was considered that there are many factors participating in the process of HCC occurrence and development, and $\mathrm{HBV}$ regulate gene expression to promote tumorigenesis by enhancing promoter activity (Ling et al., 2013; Xu et al., 2014; Yao et al., 2011). In this study, we found that DKK1 expression was associated with HBV in HCC cells, suggesting that DKK1 expression was regulated by another pathway in some HCC cases. DKK1 was reported as an inhibitor of Wnt signaling pathway and overexpression of DKK1 was reported in hepato-

blastoma (Patil et al., 2005; Yamashita et al., 2008), implying that DKK1 functions as a potential oncogenic factor in these tumors, and not as a tumor suppressor through Wnt signaling inhibition. However, the relationship between DKK1 expression and HBV and whether HBV could regulate DKK1 expression have not been studied.

In this paper, we pointed out that HBV could upregulate DKK1 expression at mRNA and protein levels. Furthermore, we found that DKK1 expression increased in cells which are transfected with pCMV-Sport6-HBx, while the rest of the plasmids encoding viral proteins (HBs, HBc, HBp) had no remarkable effects on DKK1 expression. To elucidate the mechanism of upregulation of the DKK1 expression in HCC by HBV, a series of tests on DKK1 promoter was performed. According to the results, we found HBV could upregulate DKK1 expression through activating its promoter. Moreover, transcription factors which could potentially bind to DKK1 promoter were screened and results indicated that SP1 induced DKK1 expression in a dose dependent manner. The expression increase was caused by binding to SP1 site in DKK1 promoter. SP1 functions as a transcription factor binding to the promoters of its target genes and is assumed to play pivotal role in regulating the expression of genes involved in cell proliferation, cell cycle regulation, apoptosis, migration and invasion (Hirose \& Horvitz, 2013; Karlseder et al., 1996; Black et al., 1999). More and more evidences were found that SP1 expression was abnormal in varieties of tissues, including breast, thyroid, stomach and lung (Abdelrahim et al., 2002; Chiefari et al., 2002; Wang et al., 2003; Li \& Davie, 2010). The data obtained from dual-luciferase reporter assays and Western blot indicated that the DKK1 expression declined when SP1 expression was disturbed. To further indentify the role of SP1, we removed the region containing three SP1 binding sites (nt -239 to nt -39 ) in DKK1 promoter. We found that the DKK1 promoter activity and protein expression decreased when SP1 binding sites were mutated, which suggested SP1 binding site at positions nt -239 to $n t-39$ was essential for transcriptional activation of DKK1 promoter. In conclusion, we demonstrated that transcription factor SP1 and HBV cooperated in increasing DKK1 expression in HCC. Our present data showed that HBV and SP1 both participated in regulating DKK1 expression, at the same time, we used qRT-PCR and Western blot to detect SP1 expression in order to understand whether HBV could regulate DKK1 expression in HCC. However, we failed to determine, if $\mathrm{HBV}$ has much influence on regulating SP1 expression.

In summary, we confirmed that HBV could upregulate DKK1 expression via enhancing promoter activity, and that transcription factor SP1 further increased DKK1 expression when HBV was present. DKK1 was regarded as an important factor in HCC in previous studies (Tung et al., 2011; Yu et al., 2009), and we found that HBV upregulate DKK1 expression, which further promotes the occurrence and development of HCC.

\section{Acknowledgements}

This work was supported by the Major National S\&T Program (2013ZX10002002, ALH), the major project of Chongqing Science \& Technology Commission (cstc2013jcyjC10002, ALH), the Natural Science Foundation Project of CQ CSTC (2010BB5359), and the Scientist Culture Plan of Chongqing Medical University (162014). 


\section{REFERENCES}

Abdelrahim M, Samudio I, Smith R, Burghardt R, Safe S (2002) Small inhibitory RNA duplexes for Sp1 mRNA block basal and estrogen-induced gene expression and cell cycle progression in MCF-7 breast cancer cells. J Biol Chem 277: 28815-28822. doi: 10.1074/jbc. M203828200

Black AR, Jensen D, Lin SY, Azizkhan JC (1999) Growth/cell cycle regulation of Sp1 phosphorylation. I Biol Chem 4: 1207-1215. doi: 10.1074/jbc.274.3.1207

Chiefari E, Brunetti A, Arturi F, Bidart JM, Russo D, Schlumberger M, Filetti S (2002) Increased expression of AP2 and Sp1 transcription factors in human thyroid tumors: a role in NIS expression regulation? BMC Cancer 2: 35. doi: 10.1186/1471-2407-2-35

Cougot D, Neuveut C, Buendia MA (2005) HBV induced carcinogenesis. J Clin Virol 1: S75-S78. doi: http://dx.doi.org/10.1016/S13866532(05) 80014-9

El-Serag HB, Rudolph KL (2007) Hepatocellular carcinoma: epidemiology and molecular carcinogenesis. Gastroenterology 132: 2557-2576. doi: http://dx.doi.org/10.1053/j.gastro.2007.04.061

Fedi P, Bafi co A, Nieto SA, Burgess WH, Miki T, Bottaro DP, Kraus $\mathrm{MH}$, Aaronson SA (1999) Isolation and biochemical characterization of the human Dkk-1 homologue, a novel inhibitor of mammalian Wnt signaling. J Biol Chem 274: 19465-19472. doi: 10.1074/ jbc.274.27.19465

Forget MA, Turcotte S, Beauseigle D, Godin-Ethier J, Pelletier S, Martin J, Tanguay S, Lapointe R (2007) The Wnt pathway regulator DKK1 is preferentially expressed in hormone-resistant breast tumours and in some common cancer types. Br J Cancer 96: 646-653. doi: $10.1038 /$ sj.bjc. 6603579

Glinka A, Wu W, Delius H, Monaghan AP, Blumenstock C, Niehrs C (1998) Dickkopf-1 is a member of a new family of secreted proteins and functions in head induction. Nature 391: 357-362. doi: $10.1038 / 34848$

González-Sancho JM, Aguilera O, Garcia JM, Pendás-Franco N, Peña C, Cal S, García de Herreros A, Bonilla F, Muñoz A (2005) The Wht antagonist DICKKOPF-1 gene is a downstream target of betacatenin/TCF and is downregulated in human colon cancer. Oncogene 24: 1098-1103. doi: 10.1038/sj.onc.1208303

Hirose T, Horvitz HR (2013) An Sp1 transcription factor coordinates caspase-dependent and -independent apoptotic pathways. Nature 500: 354-358. doi: 10.1038/nature12329

Karlseder J, Rotheneder H, Wintersberger E (1996) Interaction of Sp1 with the growth and cell cycle-regulated transcription factor E2F. Mol Cell Biol 16: 1659-1667. doi: 10.1128/MCB.16.4.1659

Keng VW, Tschida BR, Bell JB, Largaespada DA (2011) Modeling hepatitis $\mathrm{B}$ virus $\mathrm{X}$-induced hepatocellular carcinoma in mice with the sleeping beauty transposon system. Hepatology 53: 781-790. doi: 10.1002/hep. 24091

Krupnik VE, Sharp JD, Jiang C, Robison K, Chickering TW, Amaravadi L, Brown DE, Guyot D, Mays G, Leiby K, Chang B, Duong T, Goodearl AD, Gearing DP, Sokol SY, McCarthy SA (1999) Functional and structural diversity of the human Dickkopf gene family. Gene 238: 301-313. doi: 10.1016/S0378-1119(99)00365-0

Li L, Davie JR (2010) The role of Sp1 and Sp3 in normal and cancer cell biology. Ann Anat. 192:275-283. doi: 10.1016/j.aanat.2010.07.010

Ling Y, Zhu J, Gao L, Liu Y, Zhu C, Li R (2013) The silence of MUC2 mRNA induced by promoter hypermethylation associated with HBV in hepatocellular carcinoma. BMC Med Genet 14: 14. doi: 10.1186/1471-2350-14-14

Liu F, Pore N, Kim M, Voong KR, Dowling M, Maity A, Kao GD (2006) Regulation of histone deacetylase 4 expression by the
SP family of transcription factors. Mol Biol Cell 17: 585-597. doi: 10.1091/mbc.E05-08-0775

Livak KJ, Schmittgen TD (2001) Analysis of relative gene expression data using real-time quantitative PCR and the $2(-\Delta \Delta \mathrm{C}(\mathrm{T}))$ method. Methods 25: 402-408. doi:10.1006/meth.2001.1262

MacDonald BT, Tamai K, He X (2009) Wnt/ $\beta$-catenin signaling: components, mechanisms, and diseases. Dev Cell 17: 9-26. doi: 10.1016/j.devcel.2009.06.016

Ng SA, Lee C (2011) Hepatitis B virus X gene and hepatocarcinogenesis. J Gastroenterol 46: 974-990. doi: 10.1007/s00535-011-0415-9

Patil MA, Chua MS, Pan KH, Lin R, Lih CJ, Cheung ST, Ho C, Li R, Fan ST, Cohen SN, Chen X, So S (2005) An integrated data analysis approach to characterize genes highly expressed in hepatocellular carcinoma. Oncogene 24: 3737-3747. doi: 10.1038/ sj.onc. 1208479

Schutte K, Bornschein J, Malfertheiner P (2009) Hepatocellular carcinoma epidemiological trends and risk factors. Dig Dis 27: 80-92. doi: $10.1159 / 000218339$

Tao YM, Liu Z, Liu HL (2013) Dickkopf-1 (DKK1) promotes invasion and metastasis of hepatocellular carcinoma. Dig Liver Dis 45: 251-257. doi: 10.1016/j.dld.2012.10.020

Tian YY, Hu Y, Wang ZC, Chen K, Zhang L, Wang LY, Ren M, Huang A, Tang H (2011) Hepatitis B virus regulates Raf1 expression in HepG2.2.15 cells by enhancing its promoter activity. Arch Virol. 156: 869-874. doi: 10.1007/s00705-010-0901-z

Tung EK, Mak CK, Fatima S, Lo RC, Zhao H, Zhang C, Dai H, Poon RT, Yuen MF, Lai CL, Li JJ, Luk JM, Ng IO (2011) Clinicopathological and prognostic significance of serum and tissue Dickkopf-1 levels in human hepatocellular carcinoma. Liver Int 31: 1494-1504. doi: 10.1111/j.1478-3231.2011.02597.x

Wang L, Wei D, Huang S, Peng Z, Le X, Wu TT, Yao J, Ajani J, Xie $\mathrm{K}$ (2003) Transcription factor Sp1 expression is a significant predictor of survival in human gastric cancer. Clin Cancer Res 9: 6371-6380

Wirths O, Waha A, Weggen S, Schirmacher P, Kühne T, Goodyer CG, Albrecht S, Von Schweinitz D, Pietsch T. (2003) Overexpression of human Dickkopf-1, an antagonist of wingless/WNT signaling, in human hepatoblastomas and Wilms' tumors. Lab Invest. 83:429-434

Xu C, Zhou W, Wang Y, Qiao L (2014) Hepatitis B virus-induced hepatocellular carcinoma. Cancer Lett 345: 216-222. doi: 10.1016/j. canlet.2013.08.035

Yamashita T, Forgues M, Wang W, Kim JW, Ye Q, Jia H, Budhu A, Zanetti KA, Chen Y, Qin LX, Tang ZY, Wang XW (2008) Ep$\mathrm{CAM}$ and alpha-fetoprotein expression defines novel prognostic subtypes of hepatocellular carcinoma. Cancer Res 68: 1451-1461. doi: 10.1158/0008-5472.CAN-07-6013

Yao M, Yao DF, Bian YZ, Zhang CG, Qiu LW, Wu W, Sai WL, Yang JL, Zhang HJ (2011) Oncofetal antigen glypican-3 as a promising early diagnostic marker for hepatocellular carcinoma. Hepatobiliary Pancreat Dis Int 10: 289-294

Yu B, Yang X, Xu Y, Yao G, Shu H, Lin B, Hood L, Wang H, Yang S, Gu J, Fan J, Qin W (2009) Elevated expression of DKK1 is associated with cytoplasmic/nuclear beta-catenin accumulation and poor prognosis in hepatocellular carcinomas. J Hepatol 50: 948-957. doi: 10.1016/j.jhep.2008.11.020

Zhou Y, Wang S, Ma JW, Zhang L, Zhu HF, Lei P, Yang ZS, Zhang B, Yao XX, Shi C, Sun LF, Wu XW, Ning Q, Shen GX, Huang B (2010) Hepatitis B virus protein X-induced expression of the CXC chemokine IP-10 is mediated through activation of NF- kappaB and increases migration of leukocytes. I Biol Chem 285: 12159-12168. doi: 10.1074/jbc.M109.067629 